\title{
Association Between Metabolic Syndrome and Carotid Atherosclerosis: A Community-Based Study in Hong Kong
}

\author{
Xin Yi Leng, PhD, ${ }^{1}$ Xiang Yan Chen, PhD, ${ }^{1}$ Ping Chook, MD, ${ }^{1}$ Li Xiong, PhD, ${ }^{1}$ Wen Hua Lin, PhD, ${ }^{1}$ \\ Jing Yi Liu, PhD, ${ }^{1}$ Brian Tomlinson, MD, ${ }^{1}$ G. Neil Thomas, PhD, ${ }^{2}$ Tai Hing Lam, MD, ${ }^{3}$ \\ Karen S.L. Lam, MD, ${ }^{3}$ Bernard M.Y. Cheung, MD, PhD, ${ }^{3}$ and Ka Sing Wong, MD ${ }^{1}$
}

\section{Abstract}

Background: Carotid atherosclerosis should not be neglected as a cause for stroke in China, despite its low prevalence. This study was performed to evaluate the association between ultrasonographic markers for different stages of carotid atherosclerosis and metabolic syndrome.

Methods: This was a community-based study in Hong Kong. Metabolic syndrome was defined as having three or more of the following conditions or receiving specific treatment for these conditions: Abdominal obesity, elevated triglycerides, low high-density lipoprotein cholesterol, hypertension, and impaired fasting blood glucose. All subjects underwent carotid duplex ultrasonography. Mean carotid intima media thickness (CIMT) of bilateral common carotid arteries was used as the CIMT value for a single subject. CIMT within the $4^{\text {th }}$ quartile was regarded as increased CIMT. Carotid plaque was defined as a focal CIMT of $>1.5 \mathrm{~mm}$. A carotid plaque obstructing $\geq 50 \%$ of vessel lumen was considered as carotid stenosis.

Results: A total of 653 subjects (mean age $55.1 \pm 10.4 ; 47.2 \%$ male) were recruited. Metabolic syndrome was found in $188(28.8 \%)$ subjects (30.8\% in males and $27.0 \%$ in females). Mean CIMT was $0.74 \pm 0.12 \mathrm{~mm}$. Increased CIMT, carotid plaque, and carotid stenosis were detected in $163(25.0 \%), 95(14.5 \%)$, and $6(1.4 \%)$ subjects, respectively. In multivariate linear regression, CIMT significantly increased with increasing numbers of metabolic syndrome components $(P<0.001)$. In multivariate logistic regression analysis, metabolic syndrome was independently associated with increased CIMT [odds ratio (OR) 1.17; 95\% confidence interval (CI) 1.11-2.64; $P=0.014$ ), but not presence of carotid plaque (OR 1.50; 95\% CI 0.92-2.46; $P=0.108$ ).

Conclusions: Metabolic syndrome may be independently associated with the early stage but not the later and advanced stages of carotid atherosclerosis in community residents in China.

\section{Introduction}

$\mathrm{T}$ HERE ARE RACIAL DIFFERENCES in predilection of location of cervicocerebral arterial atherosclerosis between Caucasians and Asians. ${ }^{1}$ Despite a relatively lower prevalence in Asians than that in Caucasians, carotid atherosclerosis should not be neglected in Asian populations as a cause for ischemic stroke and transient ischemic attack, which has been increasing during the recent years. ${ }^{2-6}$ Carotid intima media thickness (CIMT), carotid plaque, and carotid stenosis determined by noninvasive ultrasonographic assessment are markers for carotid atherosclerosis and reflect different stages and aspects of atherosclerosis of the carotid arteries.
Increased CIMT mainly represents diffuse hypertensive hypertrophic response of smooth muscle cells, which is the early stage of carotid atherosclerosis, whereas the presence of carotid plaque and carotid stenosis reflects later and advanced stages of the atherosclerotic process. $^{7-9}$

Metabolic syndrome, ${ }^{10}$ a cluster of metabolic factors that includes abdominal obesity, elevated triglycerides (TG), low high-density lipoprotein cholesterol (HDL-C), hypertension, and impaired fasting blood glucose (FBG), has been found to be a predictor for different stages of carotid atherosclerosis in large studies in Caucasians. ${ }^{11-14}$ According to a cross-sectional study (2000-2001) performed in 15,540 subjects aged 35-74 years, a large proportion of Chinese

\footnotetext{
${ }^{1}$ Department of Medicine and Therapeutics, The Chinese University of Hong Kong, Shatin, Hong Kong SAR, China.

${ }^{2}$ Department of Public Health, Epidemiology and Biostatistics, University of Birmingham, Birmingham, United Kingdom.

${ }^{3}$ Li Ka Shing Faculty of Medicine, The University of Hong Kong, Pokfulam, Hong Kong SAR, China.
} 
adults have metabolic syndrome, with a prevalence of $13.7 \%$ [95\% confidence interval (CI), $12.9-14.5 \%] .{ }^{15}$ Metabolic syndrome incidence is still increasing with changes of lifestyles in China.

In the present study, we evaluated the association between metabolic syndrome and ultrasound-detected markers of carotid atherosclerosis in community-dwelling subjects in Hong Kong and tried to explore reasons for the seemingly paradoxical coexistence of the high prevalence of metabolic syndrome and low prevalence of carotid stenosis in China.

\section{Methods}

\section{Subjects}

The Hong Kong Cardiovascular Risk Factor Prevalence Study (CRISPS) was a community-based prospective cohort study of cardiovascular risk factors in Hong Kong Chinese. ${ }^{16,17}$ The study was approved by the Institutional Review Board of the University of Hong Kong/Hospital Authority Hong Kong West Cluster, and all subjects provided written informed consent prior to participation. Subjects of CRISPS-3 (2005-2008) recruited between April, 2007, and September, 2008, were screened. Those who underwent carotid duplex ultrasonography (CD) and had no missing values for metabolic syndrome components as mentioned below were enrolled in the present cross-sectional study. Smoking habits and histories of common vascular risk factors and medications were collected. Those who had ever smoked, no matter whether they quit or not, were regarded as ever-smokers. Fasting blood was analyzed for FBG, TG, HDL-C, low-density lipoprotein cholesterol (LDL-C), and total cholesterol (TC).

\section{Metabolic syndrome}

Metabolic syndrome was defined according to the US National Cholesterol Education Program (NCEP) criteria. ${ }^{10}$ Subjects with three or more of the following conditions were regarded as having metabolic syndrome: (1) Abdominal obesity (waist circumference $\geq 90 \mathrm{~cm}$ for Asian men or $\geq 80 \mathrm{~cm}$ for Asian women); (2) elevated TG levels ( $\geq 1.7$ $\mathrm{mmol} / \mathrm{L})$; (3) low HDL-C levels $(<1.03 \mathrm{mmol} / \mathrm{L}$ in men or $<1.29 \mathrm{mmol} / \mathrm{L}$ in women); (4) hypertension (blood pressure $\geq 130 / 85 \mathrm{mmHg}$ or known hypertension); and (5) impaired FBG levels ( $\geq 5.6 \mathrm{mmol} / \mathrm{L}$ or known diabetes mellitus). Receiving specific treatment for a criterion was counted as fulfilling the criterion.

\section{CD protocol}

CD was performed by an experienced technician using a Terason $\mathrm{t} 3000$ Ultrasound System (Terason $^{\mathrm{TM}}$, Burlington, MA) with a 7-MHz high-resolution linear-array transducer. Longitudinal B-mode images showing the greatest distance between the lumen-intima interface and the media-adventitia interface were stored for subsequent offline analysis, which was performed by another technician unaware of the patients' clinical data, using semiautomatic, computerized edge-detection and measurement software MATH (version 3.0, METRIS Co., Argenteuil, France). CIMT was measured within a plaque-free region with a length of at least $10 \mathrm{~mm}$, at the far wall of common carotid artery (CCA) proximal to the bifurcation. ${ }^{18}$ Mean CIMT of bilateral CCAs was regarded as the CIMT value for a single subject. A CIMT value within the $4^{\text {th }}$ quartile $\left(>75^{\text {th }}\right.$ percentile) was considered as increased CIMT.

Carotid plaque was detected in bilateral CCAs, bifurcations, and the extracranial portion of internal carotid arteries (ICA) and was defined as the presence of focal wall thickening at least $50 \%$ greater than that of the surrounding vessel wall, or a focal CIMT greater than $1.5 \mathrm{~mm}$ protruding into the lumen. ${ }^{18}$ A carotid plaque obstructing $\geq 50 \%$ of vessel lumen of CCAs, bifurcations, or extracranial ICAs was considered as carotid stenosis.

\section{Statistical analysis}

Demographic characteristics, metabolic syndrome and its components, and other vascular risk factors were compared between those with or without increased CIMT, and those with or without carotid plaques, using the Pearson chisquared test, trend test, or independent samples $t$-test. Mean CIMT values of subjects with different numbers of metabolic syndrome components were compared using one-way analysis of variance (ANOVA). A linear regression model was built to evaluate the linear relationship between the number of metabolic syndrome components and CIMT values. Logistic regression models were built to calculate adjusted odds ratios (ORs) of the presence of metabolic syndrome and the number of its components for the presence of increased CIMT and carotid plaque, respectively. All statistical analyses were performed in PASW Statistics (version 18.0, IBM SPSS Statistics, Chicago, IL). Two-sided $P$ values of $<0.05$ were considered to be statistically significant.

\section{Results}

A total of 653 community-dwelling subjects (mean age $55.1 \pm 10.4 ; 47.2 \%$ male) were recruited. An overview of demographic characteristics, metabolic syndrome components, and other vascular risk factors is shown in Table 1. Overall, metabolic syndrome was found in 188 (28.8\%) subjects (30.8\% in male and $27.0 \%$ in female), with $124(19.0 \%), 196$ $(30.0 \%), 145(22.2 \%), 115(17.6 \%), 51(7.8 \%)$, and $22(3.4 \%)$ subjects having zero to five components, respectively. Abdominal obesity, elevated TG, low HDL-C, hypertension, and impaired FBG were found in 285 (43.6\%), 140 (21.4\%), $266(40.7 \%), 312(47.8 \%)$, and $142(21.7 \%)$ subjects, respectively. Mean CIMT was $0.74 \pm 0.12 \mathrm{~mm}$. The $75^{\text {th }}$ percentile of CIMT values was $0.795 \mathrm{~mm}$, and 163 (25.0\%) subjects had increased CIMT. Carotid plaques were detected in 95 (14.5\%) subjects (62 males and 33 females), among whom only 6 $(1.4 \%)$ had carotid stenosis.

\section{Association between metabolic syndrome and CIMT}

Mean CIMT values were significantly increased with increasing numbers of metabolic syndrome components $(P<0.001$ for one-way ANOVA; Fig. 1). Multivariate linear regression model revealed that the number of metabolic syndrome components was independently related to CIMT values (beta $=0.015$ per one component increase with constant age, gender, ever-smoker, TC, and LDL-C; $P<0.001)$. 
Table 1. Overview of Characteristics and Univariate Analysis for Subjects with/without Increased Cimt or Presence of Carotid Plaque

\begin{tabular}{|c|c|c|c|c|c|c|c|}
\hline \multirow[b]{2}{*}{ Characteristics } & \multirow[b]{2}{*}{ Overall $(\mathrm{N}=653)$} & \multicolumn{3}{|c|}{ Increased CIMT } & \multicolumn{3}{|c|}{ Presence of carotid plaque } \\
\hline & & Yes $(\mathrm{n}=163)$ & No $(\mathrm{n}=490)$ & $\mathrm{P}$ & Yes $(\mathrm{n}=95)$ & No $(\mathrm{n}=558)$ & $\mathrm{P}$ \\
\hline Age & $55.1(10.4)$ & $63.3(9.7)$ & $52.4(9.1)$ & $<0.001$ & $64.2(10.4)$ & $53.5(9.5)$ & $<0.001$ \\
\hline Male & $308(47.2)$ & $99(60.7)$ & $209(42.7)$ & $<0.001$ & $62(65.3)$ & $246(44.1)$ & $<0.001$ \\
\hline Ever-smoker & $188(28.8)$ & $58(35.6)$ & $130(26.5)$ & 0.027 & $42(44.2)$ & $146(26.2)$ & $<0.001$ \\
\hline TC (mmol/L) & $4.92(1.08)$ & 5.09 (1.12) & $4.86(1.06)$ & 0.017 & 4.97 (1.17) & $4.91(1.06)$ & 0.607 \\
\hline LDL-C (mmol/L) & $3.02(0.85)$ & $3.19(0.90)$ & $2.96(0.83)$ & 0.004 & $3.07(0.94)$ & $3.01(0.84)$ & 0.511 \\
\hline Presence of metabolic syndrome & $188(28.8)$ & $71(43.6)$ & $117(23.9)$ & $<0.001$ & $42(44.2)$ & $146(26.2)$ & $<0.001$ \\
\hline \multicolumn{8}{|l|}{ Metabolic syndrome components } \\
\hline Abdominal obesity & $285(43.6)$ & $83(50.9)$ & $202(41.2)$ & 0.031 & $44(46.3)$ & $241(43.2)$ & 0.570 \\
\hline Elevated TG & $140(21.4)$ & $46(28.2)$ & $94(19.2)$ & 0.015 & $31(32.6)$ & $109(19.5)$ & 0.004 \\
\hline Low HDL-C & $266(40.7)$ & $73(44.8)$ & $193(39.4)$ & 0.224 & $47(49.5)$ & $219(39.2)$ & 0.061 \\
\hline Hypertension & $312(47.8)$ & $115(70.6)$ & $197(40.2)$ & $<0.001$ & $67(70.5)$ & $245(43.9)$ & $<0.001$ \\
\hline Impaired FBG & $142(21.7)$ & $62(38.0)$ & $80(16.3)$ & $<0.001$ & $39(41.1)$ & $103(18.5)$ & $<0.001$ \\
\hline \multicolumn{8}{|c|}{ Number of metabolic syndrome components } \\
\hline 0 & $124(19.0)$ & $11(6.7)$ & $113(23.1)$ & $<0.001^{\mathrm{b}}$ & $7(7.4)$ & $117(21.0)$ & $<0.001^{\mathrm{b}}$ \\
\hline 1 & $196(30.0)$ & $36(22.1)$ & $160(32.7)$ & & $22(23.2)$ & $174(31.2)$ & \\
\hline 2 & 145 (22.2) & 45 (27.6) & $100(20.4)$ & & $24(25.3)$ & 121 (21.7) & \\
\hline 3 & 115 (17.6) & $41(25.2)$ & 74 (15.1) & & $21(22.1)$ & $94(16.8)$ & \\
\hline 4 & $51(7.8)$ & $20(12.3)$ & 31 (6.3) & & $10(10.5)$ & $41(7.3)$ & \\
\hline 5 & $22(3.4)$ & $10(6.1)$ & $12(2.4)$ & & 11 (11.6) & $11(2.0)$ & \\
\hline
\end{tabular}

${ }^{a}$ Values are means (standard deviation, SD) or numbers (\%).

${ }^{\mathrm{b}} P$ for trend.

CIMT, carotid intima-media thickness; TC, total cholesterol; LDL-C, low-density lipoprotein cholesterol; TG, triglycerides; HDL-C, highdensity lipoprotein cholesterol; FBG, impaired fasting blood glucose.

In univariate analysis, subjects with increased CIMT were more likely to have metabolic syndrome $(43.6 \%$ versus $23.9 \%, P<0.001)$, and they also tended to have more metabolic syndrome components $(P<0.001$ for trend; Table 1 and Fig. 2). In addition, more of those with increased CIMT had

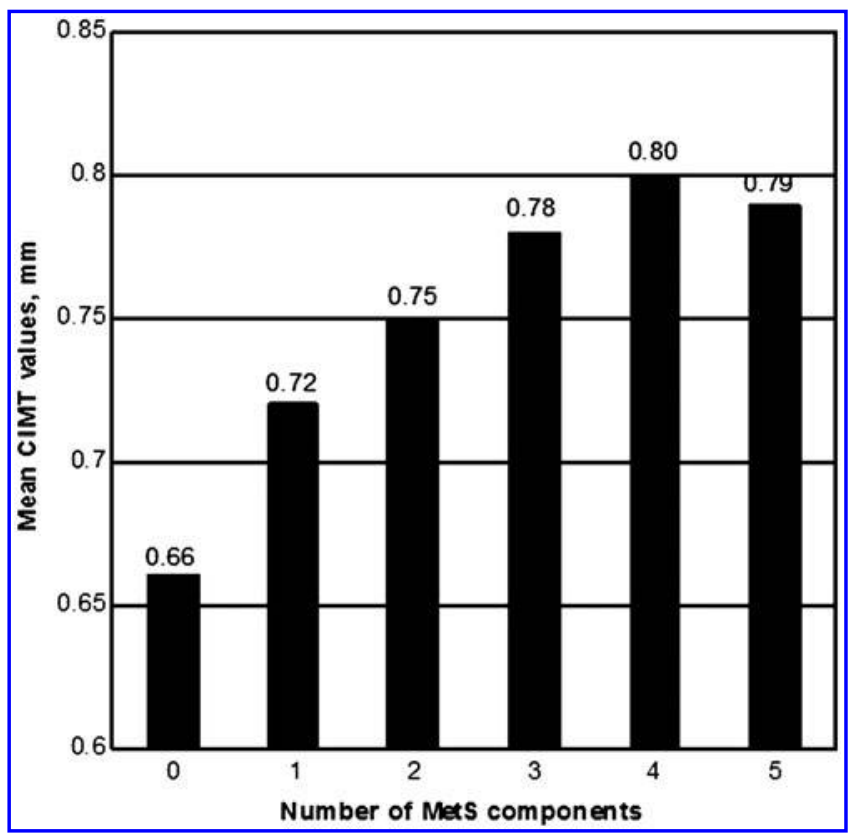

FIG. 1. Mean carotid intima media thickness (CIMT) values were significantly increased with increasing numbers of metabolic syndrome components $[P<0.001$ for one-way analysis of variance (ANOVA)]. MetS, metabolic syndrome. abdominal obesity ( $50.9 \%$ versus $41.2 \%, P=0.031)$, elevated TG $(28.2 \%$ versus $19.2 \%, P=0.015)$, hypertension $(70.6 \%$ versus $40.2 \%, P<0.001)$, and impaired FBG $(38.0 \%$ versus $16.3 \%, P<0.001$ ). In multivariate logistic regression (Table 2 ), the presence of metabolic syndrome was independently

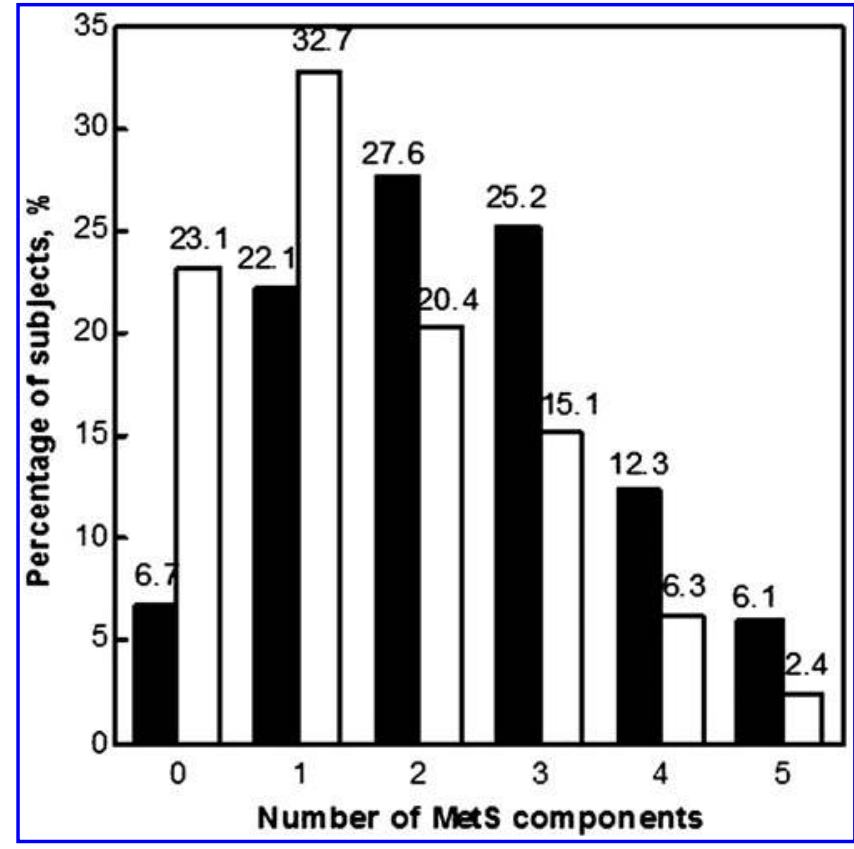

FIG. 2. Subjects with increased carotid intima media thickness (CIMT) had more metabolic syndrome components $(P<0.001$ for trend). (black bars) Subjects with increased CIMT; (white bars) subjects without increased CIMT. MetS, metabolic syndrome. 
Table 2. Results for Multivariate Logistic Regression Models

\begin{tabular}{|c|c|c|c|c|}
\hline \multirow[b]{2}{*}{ Characteristics } & \multicolumn{2}{|c|}{ Increased $\mathrm{CIMT}^{\mathrm{a}}$} & \multicolumn{2}{|c|}{ Presence of carotid plaque } \\
\hline & Adjusted ORs (95\% CI) & $\mathrm{P}$ & Adjusted ORs $(95 \%$ CI) & $\mathrm{P}$ \\
\hline Presence of metabolic syndrome & $1.72(1.11-2.64)$ & 0.014 & $1.50(0.92-2.46)$ & 0.108 \\
\hline \multicolumn{5}{|c|}{ Number of metabolic syndrome components } \\
\hline 0 & 1.00 & & 1.00 & \\
\hline 1 & $1.61(0.73-3.55)^{\mathrm{c}}$ & 0.240 & $1.40(0.55-3.58)^{\mathrm{c}}$ & 0.482 \\
\hline 2 & $2.47(1.12-5.45)^{\mathrm{c}}$ & 0.026 & $1.61(0.63-4.12)^{\mathrm{c}}$ & 0.324 \\
\hline 3 & $3.02(1.35-6.78)^{\mathrm{c}}$ & 0.007 & $1.72(0.66-4.50)^{\mathrm{c}}$ & 0.267 \\
\hline 4 & $2.88(1.12-7.41)^{\mathrm{c}}$ & 0.028 & $1.58(0.52-4.81)^{\mathrm{c}}$ & 0.420 \\
\hline 5 & $3.52(1.05-11.76)^{c}$ & 0.041 & $7.92(2.30-27.33)^{c}$ & 0.001 \\
\hline
\end{tabular}

${ }^{a}$ Adjusting for age, male gender, ever-smoker, TC, and LDL-C.

${ }^{\mathrm{b}}$ Adjusting for age, male gender and ever-smoker.

CCompared with those with no metabolic syndrome components.

CIMT, carotid intima-media thickness; OR, odds ratio; CI, confidence interval; TC, total cholesterol; LDL-C, low-density lipoprotein cholesterol.

related to increased CIMT (OR 1.17; 95\% CI 1.11-2.64; $P=0.014)$, after adjusting for age, male gender, ever-smoker, TC, and LDL-C. Risks of having increased CIMT significantly increased for those with two, three, four, or five metabolic syndrome components, compared with those without a metabolic syndrome component (all $P$ values $<0.05)$.

\section{Association between metabolic syndrome and presence of carotid plaque}

In univariate analysis (Table 1), subjects with carotid plaques had a significantly higher incidence of metabolic syndrome, compared with those had no carotid plaque (44.2\% versus $26.2 \%, P<0.001)$. They also had more metabolic syndrome components $(P<0.001$ for trend; Fig. 3$)$, and more of them had elevated TG $(32.6 \%$ versus $19.5 \%$, $P=0.004)$, hypertension $(70.5 \%$ versus $43.9 \%, P<0.001)$, and impaired FBG (41.1\% versus $18.5 \%, P<0.001)$. However, metabolic syndrome was not independently related to the presence of carotid plaque (OR 1.50; 95\% CI 0.92-2.46; $P=0.108$ ) after adjusting for age, male, and ever-smoker. Compared with those without a metabolic syndrome component, only those with five components (OR 7.92; 95\% CI 2.30-27.33; $P=0.001$ ) had significantly higher risk of having carotid plaque (Table 2). The incidence of carotid stenosis was too low $(1.4 \%)$ in this study to analyze its association with metabolic syndrome.

\section{Discussion}

In the present study, we found a high prevalence of metabolic syndrome $(28.8 \%)$ in community residents in Hong Kong SAR, China. CIMT significantly increased with increasing numbers of metabolic syndrome components. Also, the presence of metabolic syndrome and the number of its components were independent predictors for increased CIMT. However, metabolic syndrome was not independently related to presence of carotid plaque.

The prevalence of metabolic syndrome $(30.8 \%$ in male and $27.0 \%$ in female) in our study was comparable to that in the United States in 1999-2002, which was about 33.7\% among men and $35.4 \%$ among women, based on the NCEP definition, ${ }^{10}$ the same that used in this study except for the crite- rion of abdominal obesity (102 and $88 \mathrm{~cm}$ for American males and females, respectively). ${ }^{19}$

According to the results, metabolic syndrome was independently associated with increased CIMT, which represents an early stage of carotid atherosclerosis, but no significant correlation existed between metabolic syndrome and the presence of carotid plaque, the later stage of carotid atherosclerosis. This was similar to a Japanese study performed in 1540 symptomatic subjects, with metabolic syndrome independently related to CIMT $>1.10 \mathrm{~mm}$ but not plaque occurrence. ${ }^{20}$ Our results were not in accordance with the results of a population-based study with a large sample size (6142 subjects) in eastern China, in which metabolic syndrome and

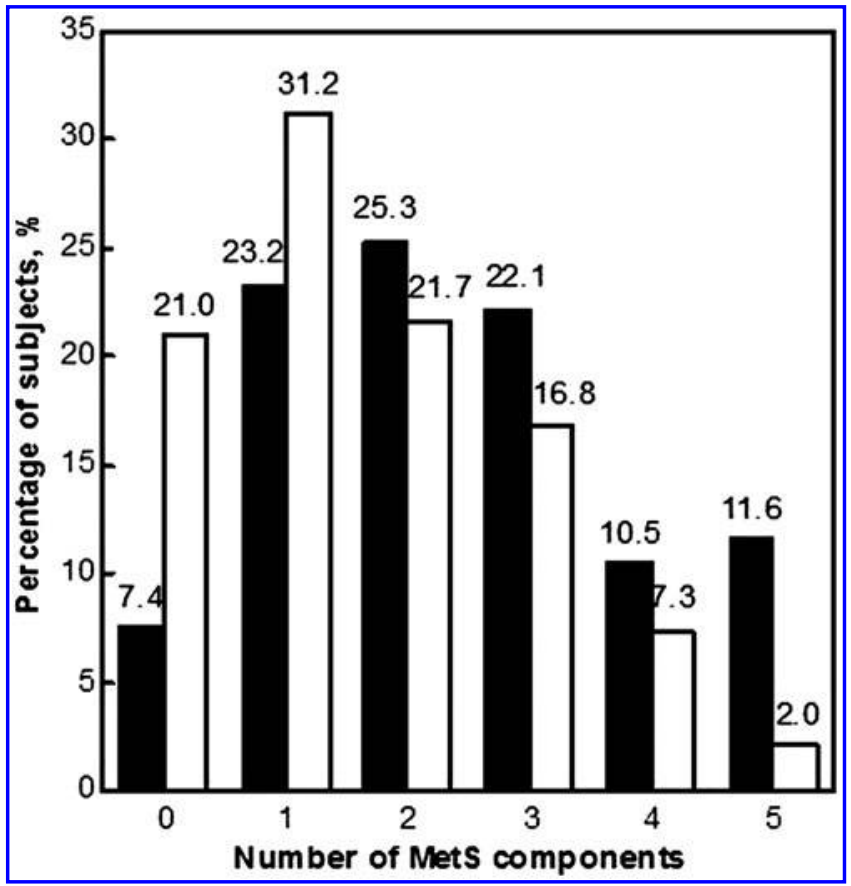

FIG. 3. Subjects with carotid plaques had more metabolic syndrome components in univariate analysis $(P<0.001$ for trend). (black bars) Subjects with carotid plaque; (white bars) subjects without carotid plaque. MetS, metabolic syndrome. 
number of its components were reported to be associated with increased CIMT and the presence of carotid plaque in univariate analysis. ${ }^{21}$ However, those authors did not provide results of multivariate analysis on these associations. It was possible that the results would be different after adjusting for confounding factors.

The incidence of carotid stenosis was too low (1.4\%) in this study for the analysis of its association with metabolic syndrome. A previous study performed among acute ischemic stroke or transient ischemic attack patients admitted to the UCLA Medical Center found that metabolic syndrome was independently related to carotid stenosis in Caucasians (OR 1.98; 95\% CI 1.13-3.45), but not in subjects with other ethnic origins, after adjusting for covariates. ${ }^{14}$ Another study found metabolic syndrome to be more associated with intracranial arterial stenosis than carotid stenosis in a Korean population. $^{22}$ Therefore, by combining the results of our study and previous studies on the association between metabolic syndrome and carotid stenosis, we determined that in populations with Asian origins, although metabolic syndrome might affect the early stage of atherogenesis of carotid arteries, later and advanced stages of carotid atherosclerosis (mainly represented as carotid plaque and carotid stenosis) are more probably due to other factors. This may partly explain the seemingly paradoxical coexistence of the high prevalence of metabolic syndrome and low prevalence of carotid stenosis in China.

The strength of our study was that we evaluated the association between metabolic syndrome and different stages of carotid atherosclerosis in community-dwelling subjects in China, which to some extent further explained the racial difference in the distribution of cervicocerebral arterial atherosclerosis between Caucasians and Asians. However, the small number of subjects with carotid stenosis made it impossible for analysis of its association with metabolic syndrome, which was a limitation of our study. Also, the numbers of male and female subjects with carotid plaques were not large enough for multivariate analysis of genderspecific associations between metabolic syndrome and carotid atherosclerosis.

In conclusion, our study indicated that metabolic syndrome might be independently associated with early-stage carotid atherosclerosis but not later and advanced stages of carotid atherosclerosis in community residents in China. Combined with results of previous studies, we also partly explained the seemingly paradoxical coexistence of the high prevalence of metabolic syndrome and low prevalence of carotid stenosis in China.

\section{Acknowledgments}

The study was supported by the National Natural Science Foundation of China (30518001/CO301070202); the Hong Kong Research Grant Council grants (\#7229/01M and \#7626/07M); Sun Chieh Yeh Heart Foundation of Hong Kong; the S. H. Ho Cardiovascular Disease and Stroke Centre, Institute of Vascular Medicine, The Chinese University of Hong Kong; and the University of Hong Kong Foundation for Educational Development and Research.

\section{Author Disclosure Statement}

No competing financial interests exist.

\section{References}

1. Wong LKS. Global burden of intracranial atherosclerosis. Int L Stroke 2006;1:158-159.

2. Nagao T, Sadoshima S, Ibayashi S, Takeya Y, Fujishima M. Increase in extracranial atherosclerotic carotid lesions in patients with brain ischemia in Japan: An angiographic study. Stroke 1994;25:766-770.

3. Liu H, Tu Y, Yip P, Su C. Evaluation of intracranial and extracranial carotid steno-occlusive diseases in Taiwan Chinese patients with MR angiography. Preliminary experience. Stroke 1996;27:650-653.

4. Huang Y, Gao S, Li S, et al. Vascular lesions in Chinese patients with transient ischemic attacks. Neurology 1997;48:524-525.

5. Woo KS, Chook P, Raitakari OT, et al. Westernization of Chinese adults and increased subclinical atherosclerosis. Arterioscler Thromb Vasc Biol 1999;19:2487-2493.

6. Dharmasaroja P. Prevalence of extracranial carotid stenosis in Thai ischemic stroke/TIA patients. I Neurol Sci 2008;269: 92-95.

7. Stary HC, Chandler AB, Dinsmore RE, et al. A definition of advanced types of atherosclerotic lesions and a histological classification of atherosclerosis. A report from the Committee on Vascular Lesions of the Council on Arteriosclerosis, American Heart Association. Arterioscler Thromb Vasc Biol 1995;15:1512-1531.

8. Spence JD. Measurement of intima-media thickness vs. carotid plaque: Uses in patient care, genetic research and evaluation of new therapies. Int J Stroke 2006;1:216-221.

9. Mathiesen EB, Johnsen SH. Ultrasonographic measurements of subclinical carotid atherosclerosis in prediction of ischemic stroke. Acta Neurol Scand 2009;120:68-72.

10. Grundy S, Brewer H, Cleeman J, et al., for the conference participants. Definition of metabolic syndrome. Report of the National Heart, Lung, and Blood Institute/American Heart Association Conference on Scientific Issues Related to Definition. Circulation 2004;109:433-438.

11. McNeill A, Rosamond W, Girman C, et al. Prevalence of coronary heart disease and carotid arterial thickening in. patients with the metabolic syndrome (The ARIC study). $\underline{A m}$ L Cardiol 2004;94:1249-1254.

12. Tzou W, Douglas P, Srinivasan S, et al. Increased subclinical atherosclerosis in young adults with metabolic syndrome. The Bogalusa Heart Study. I Am Coll Cardiol 2005;46:457463.

13. Antonini-Canterin F, La Carrubba S, Gullace G, et al. Association between carotid atherosclerosis and metabolic syndrome: results from the ISMIR study. Angiology 2010;61:443-448.

14. Bang OY, Saver JL, Liebeskind DS, et al. Impact of metabolic syndrome on distribution of cervicocephalic atherosclerosis: Data from a diverse race-ethnic group. L Neurol Sci 2009;284:40-45.

15. Gu DF, Reynolds K, Wu XG, et al. Prevalence of the metabolic syndrome and overweight among adults in China. Lancet 2005;365:1398-1405.

16. Janus E. Epidemiology of cardiovascular risk factors in Hong Kong. Clin Exp Pharmacol Physiol 1997;24:987-988.

17. Cheung BMY, Ong KL, Tso AWK, et al. Relationship of plasma interleukin-6 and its genetic variants with hypertension in Hong Kong Chinese. Am I Hypertens 2011;24: 1331-1337.

18. Touboul PJ, Hennerici MG, Meairs S, et al. Mannheim carotid intima-media thickness consensus (2004-2006). An update on behalf of the Advisory Board of the 3rd and 4th 
Watching the Risk Symposium 13th and 15th European Stroke Conferences, Mannheim, Germany, 2004, and Brussels, Belgium, 2006. Cerebrovasc Dis 2007;23:75-80.

19. Ford E. Prevalence of the metabolic syndrome defined by the International Diabetes Federation among adults in the U.S. Diabetes Care 2005;28:2745-2749.

20. Kawamoto R, Ohtsuka N, Ninomiya D, et al. Aging and metabolic syndrome effect on carotid atherosclerosis assessed by ultrasonography. Geriatr Gerontol Int 2007;7:221-228.

21. Yin JH, Song ZY, Shan $\mathrm{P}$, et al. Age- and gender-specific prevalence of carotid atherosclerosis and its association with metabolic syndrome in Hangzhou, China. Clin Endocrinol 2012;76:802-809.
22. Park JH, Kwon HM, Roh JK. Metabolic syndrome is more associated with intracranial atherosclerosis than extracranial atherosclerosis. Eur J Neurol 2007;14:379-386.

Address correspondence to: Ka Sing Wong, MD

Department of Medicine and Therapeutics The Chinese University of Hong Kong Prince of Wales Hospital Shatin, Hong Kong SAR China

E-mail: ks-wong@cuhk.edu.hk 